\title{
PROGRAMA DE INTERIORIZAÇÃO DO TRABALHO EM SAÚDE NA PARAÍBA: MUDANÇAS METODOLÓGICAS E REFLEXOS NA PRODUÇÃO ACADÊMICA DOS EGRESSOS
}

César Cavalcanti da Silva ${ }^{1}$, Ana Tereza Medeiros Cavalcanti da Silva² ${ }^{2}$ Luciana Dantas Farias ${ }^{3}$

\begin{abstract}
RESUMO: Analisou-se o Programa de Interiorização do Trabalho em Saúde (PITs), na Paraíba, durante os anos de 2002 a 2004, objetivando caracterizar as transformações pedagógico-estruturais dos três cursos de especialização promovidos pelo PITs, na Paraíba e relacionar a produção acadêmica dos egressos desses cursos com as mudanças pedagógicoestruturais verificadas nas três versões. Os cursos de especialização ocorreram no Núcleo de Estudos em Saúde Coletiva (NESC) do Centro de Ciências da Saúde da Universidade Federal da Paraíba (CCS/UFPB). Procedeu-se a um levantamento da estrutura acadêmica dos três cursos, com uma revisão no número de disciplinas, cargas horárias e produção científica dos egressos de cada curso. Com base na análise das mudanças pedagógico-estruturais nas três edições do curso concluiu-se que houve uma correspondência direta entre as mudanças pedagógico-estruturais dos cursos e a opção pelo tema da monografia escolhido pelos egressos.
\end{abstract}

PALAVRAS-CHAVE: Recursos Humanos; Trabalho; Saúde

\section{INTERIORIZATION PROGRAMME OF HEALTH WORK IN PARAÍBA STATE, BRAZIL: METHODOLOGICAL CHANGES AND REFLECTIONS ON GRADUATES’ACADEMIC PRODUCTION}

\begin{abstract}
The Internalization Program of Health Work in Paraiba State/Brazil was analyzed between 2002 and 2004 aiming to feature teaching-structural changes in the three specialization courses promoted by the program, and relate the academic production in theses courses to the teaching-structural changes verified in the three courses. The specialization courses were held in the Study Group of Collective Health from the Center of Health Sciences at the Federal University of Paraiba. A survey of the academic structure of the three courses was carried out, with a review in the number of disciplines, number of hours for each of them and undergraduates' scientific production in each course. Based on the analysis of the teaching-structural changes in the three editions of the course, it was concluded that there was a direct correlation between the teaching-structural changes in the courses and the theme option in undergraduates' monograph.
\end{abstract}

KEYWORDS: Human Resources; Work; Health.

\section{PROGRAMA DE INTERIORIZACIÓN DE TRABAJO EN SALUD EN PARAÍBA: CAMBIOS EN METODOLÓGICOS Y REFLEJOS EN LA PRODUCCIÓN ACADÉMICA DE EGRESSOS}

RESUMEN: Fue analizado en Programa de Trabajo en Salud Interiorização (PITs), en Paraíba, mientras los años 2002 a 2004 , a fin de caracterizar las transformaciones estructurales de la enseñanza de los tres cursos de especialización que se forman en el Núcleo de Estudios en Salud Pública (NESC), de Centro de Ciencias de la Salud de la Universidad Federal de Paraíba (CCS / UFPB). Hubo un levantamiento de la estructura académica de los tres cursos, con una revisión del número de disciplinas, la carga horaria y la producción científica de egressos de cada curso. Acerca de la base del análisis de los cambios estructurales en la enseñanza de tres ediciones del curso, se llegó a la conclusión de que hay una correlación directa entre los cambios estructurales de la enseñanza de cursos en el tema de la opción de monografía elegida por los egressos.

PALABRAS CLAVE: Recursos humanos; Trabajo; Salud.

\footnotetext{
${ }^{1}$ Enfermeiro. Doutor em Enfermagem. Docente do Departamento de Enfermagem Médico-Cirúgica e Administração da Universidade Federal da Paraíba-UFPB.

${ }^{2}$ Enfermeira. Doutora em Enfermagem. Docente do Departamento de Enfermagem em Saúde Pública e Psiquiatria da UFPB.

${ }^{3}$ Enfermeira. Mestre em Enfermagem pela UFPB.
}

Autor correspondente:

César Cavalcanti da Silva

Avenida Umbuzeiro, 209 - 58038-180 - João Pessoa-PB

E-mail: profccs@yahoo.com.br 


\section{INTRODUÇÃO}

No Brasil, a formação de recursos humanos em saúde tem sido reconhecida como elemento crítico no processo de reorientação e transformação dos sistemas de saúde. Essa área requer uma aproximação interdisciplinar e não tem sido tratada com a relevância política que merece.

As questões relacionadas à constituição de recursos humanos em saúde jamais deixaram de ser motivo de preocupação para formadores, trabalhadores e gestores. Essas questões aparecem com freqüência como objeto de investigação para pesquisadores, os quais, ao longo dos últimos anos, produziram importantes análises e encetaram diferentes propostas que, se houvessem sido implantadas, quer pelos formadores, quer pelos gestores, teriam minorado grande parte das distorções que hoje afetam o trabalho na saúde ${ }^{(1)}$.

Revendo a história das investigações sobre a formação de recursos humanos em saúde no Brasil, verifica-se que, entre as décadas de 50 e 60 , foi comum a ocorrência de estudos voltados para os processos de formação de trabalhadores de saúde de nível universitário, e de estudos descritivos sobre a oferta de pessoal de saúde e sua distribuição geográfica ${ }^{(2)}$.

Nas décadas de 70 e 80 , as investigações agrupadas sob a denominação de organização social das práticas de saúde não apresentaram resultados imediatamente aplicáveis, entretanto, subsidiaram estudos concretos, consolidados sob a denominação de força de trabalho em saúde que em muito contribuíram para a compreensão do conjunto de trabalhadores de saúde como um todo articulado e não mais uma somatória de técnicos ${ }^{(2)}$.

Apesar de toda a produção acadêmica, e dos inúmeros eventos relacionados ao tema formação de recursos humanos de saúde, especialmente as Conferências Nacionais de Saúde, o poder público ainda não conseguiu priorizar a implantação de uma Política Nacional que consiga dar conta dos graves problemas que atingem a essa área e interferem negativamente no processo de efetivação do Sistema Único de Saúde (SUS).

A reflexão sobre a formação de recursos humanos em saúde tem importância estratégica dadas às necessidades decorrentes da implementação do SUS e a conseqüente mudança de perfis profissionais para atender seus princípios norteadores, doutrinários e organizativos.

O SUS é uma formulação política e organizacional que visa, dentre outros objetivos, o reordenamento dos serviços e ações de saúde, estabelecida pela Constituição Federal de 1988, para tanto, segue a mesma doutrina e os mesmos princípios organizativos em todo o território nacional, sob a responsabilidade das três esferas autônomas de governo. Baseado em preceitos constitucionais, a construção do Sistema Único de Saúde segue os princípios doutrinários da universalidade, equidade e integralidade, e quanto à organização, encontra-se amparado pelos princípios da regionalização e hierarquização, resolubilidade, descentralização, participação dos cidadãos, e pela complementaridade do setor privado(3).

Salienta-se que mesmo após a promulgação da Constituição cidadã de 1988, a organização dos serviços de saúde no Brasil, manteve um perfil centralizado, com predomínio dos serviços privados, distante das reais necessidades de saúde da população.

O reconhecimento desse modelo de organização dos serviços de saúde, discriminatório e injusto, aliado às pressões dos movimentos sociais de luta em defesa da universalização da assistência e mais atenção à prevenção, do que a cura fez com que o Ministério da Saúde apresentasse em 1994, o Programa Saúde da Família (PSF) ${ }^{(4)}$.

Segundo a proposta ministerial, esse Programa deveria funcionar, sobretudo, como estratégia de reforço ao Sistema Único de Saúde desenvolvendo ações de promoção e proteção à saúde do indivíduo, da família e da comunidade, através de equipes multiprofissionais, que fariam atendimento nas comunidades e unidades básicas de saúde, atuando prioritariamente no nível primário de atenção à saúde.

O PSF surgiu para aproximar os serviços de saúde da população, cumprindo o princípio constitucional do Estado de garantir ao cidadão seu direito de receber atenção integral à saúde com prioridade para a prevenção, mas sem prejuízo dos serviços assistenciais. Para induzir os gestores municipais a organizarem suas ações de saúde e assim implantarem a estratégia do Programa Saúde da Família, o Ministério da Saúde instituiu um programa de caráter transitório, conhecido por Programa de Interiorização do Trabalho em Saúde (PITs) ${ }^{(5)}$.

O Programa de Interiorização do Trabalho em Saúde pode ser considerado, portanto, como uma estratégia desenvolvida pelo Ministério da Saúde para melhorar a qualificação profissional, bem como expandir a cobertura da assistência à saúde para a população do interior do país. Definido como a estratégia para a 
reorganização da atenção básica de saúde, o Programa foi instituído pelo Decreto ${ }^{\circ} 3.745$, de 05 de fevereiro de 2001, regulamentado pela Portaria $n^{\circ} 227$, de 16 de fevereiro de 2001, e concebido no intuito de fortalecer o Programa de Saúde da Família e, conseqüentemente, atender as populações de Municípios desprovidos ou com precários serviços médico-sanitários, com a alocação de enfermeiros e médicos em seus territórios, mediante a oferta de incentivos financeiros e de formação profissional aos participantes ${ }^{(6)}$.

Como pré-requisito para a implantação do PITs, o Município já deveria ter implantado o Programa de Agentes Comunitários de Saúde (PACS), ter população de até 50 mil habitantes, não dispor de equipe de saúde da família, apresentar altas taxas de mortalidade infantil, ser área de prioridade para o controle da malária e ou de tuberculose e de hanseníase. Outro fator relevante para a seleção era o número de consultas por habitante/ ano, e também a inclusão desses municípios em outros programas governamentais de combate à pobreza e à exclusão social ${ }^{(6)}$.

O Programa de Interiorização do Trabalho em Saúde previa a alocação de enfermeiros e médicos encarregados da redução dos níveis de adoecimento e conscientização dos cidadãos acerca de sua integração e participação no meio social, considerado como determinantes das condições de saúde. Para que as duplas de profissionais mantivessem adequados níveis de atualização profissional, além de suporte para o enfrentamento de questões ligadas à saúde, o Ministério da Saúde inseriu como parte da política de interiorização, a realização de um Curso de Especialização em Saúde da Família, além de acesso à Internet, como meio de aquisição de conhecimento e interação permanente com os especialistas dos grandes centros ${ }^{(6)}$.

O curso de especialização que adotou a estratégia do PSF como modelo de organização da atenção básica foi o processo de qualificação utilizado pelo Ministério da Saúde para inserir os profissionais em sua prática.

O apoio e a execução desses cursos ficaram a cargo dos Pólos de Capacitação, Formação e Educação Permanente de Pessoal para Saúde da Família, cujo objetivo era formar enfermeiros e médicos para desempenhar suas atividades profissionais em unidades básicas, em conformidade com a Estratégia Saúde da Família, por intermédio de ações de abordagem coletiva e clínica individual.

No Estado da Paraíba o curso de especialização em saúde da família foi oferecido pelo Núcleo de Estudos em Saúde Coletiva - NESC e teve início em julho de 2001 com a participação de 30 profissionais, sendo 16 enfermeiros e 14 médicos, oriundos de onze municípios: Cacimba de Dentro, Caldas Brandão, Carrapateira, Casserengue, Curral Velho, Diamante, Juarez Távora, Tavares, Monte Horebe, Nazarezinho e São José de Princesa. Na seqüência foram realizados outros dois cursos de especialização perfazendo um total de três turmas e a produção de vinte e nove monografias, sendo doze apresentadas em 2002, dezesseis em 2003 e apenas uma em 2004.

Considerando que a estrutura acadêmica de um curso reflete a política de saúde na qual se insere e constitui o eixo para a composição do perfil profissional da formação da força de trabalho que se agrega a esse curso, questiona-se: as mudanças estruturais na composição dos três cursos patrocinados pelo PITs, ocorridos na Paraíba, influenciaram a produção acadêmica dos egressos? Para responder a essa questão, formularam-se os seguintes objetivos: 1) Caracterizar as transformações pedagógico-estruturais dos três cursos de especialização promovidos pelo PITs, na Paraíba. 2) Relacionar a produção acadêmica dos egressos dos três cursos de especialização promovidos pelo PITs na Paraíba, com as mudanças pedagógicoestruturais verificadas nas três versões.

\section{MÉTODOS}

Trata-se de um estudo do tipo documental, realizado no Núcleo de Estudos em Saúde Coletiva do Centro de Ciências da Saúde da Universidade Federal da Paraíba. A pesquisa foi iniciada no mês de abril de 2005 e concluída no mês de junho de 2006.

Para responder ao primeiro objetivo procedeuse à primeira etapa da pesquisa que constou de um levantamento da estrutura acadêmica dos três cursos de especialização promovidos pelo PITs no Estado da Paraíba, ocorrendo o reconhecimento dos seguintes elementos constitutivos de cada estrutura: número de disciplinas, cargas horárias e a partir do segundo curso, inserção ou retirada de novas disciplinas.

Para responder ao segundo objetivo da investigação, procedeu-se à segunda etapa da pesquisa que constou de um levantamento de toda a produção científica dos egressos de cada curso, a partir do material existente na biblioteca do Núcleo de Estudos em Saúde Coletiva (NESC).

O procedimento ético foi observado segundo o item VII.14.letra a, da Resolução 196/1996(7) que dispõe sobre a atuação do Comitê de Ética em Pesquisa, e 
assevera que:

A revisão ética de toda e qualquer proposta de pesquisa envolvendo seres humanos não poderá ser dissociada da sua análise científica. Pesquisa que não se faça acompanhar do respectivo protocolo não deve ser analisada pelo Comitê.

O acesso ao acervo foi precedido do consentimento formal da Coordenadora do Núcleo de Estudos em Saúde Coletiva e comunicado oficial à bibliotecária da instituição. Os trabalhos foram catalogados por ano de apresentação e a verificação das mudanças pedagógico-estruturais na composição dos trabalhos foi feita a partir da leitura dos resumos e objetivos do estudo.

\section{APRESENTAÇÃO E DISCUSSÃO DOS DADOS}

Quadro 01 - Caracterização pedagógico-estruturais dos cursos de especialização promovidos pelo PITs na Paraíba

\begin{tabular}{|c|c|c|}
\hline $\begin{array}{c}\text { Grade Curricular e Carga horária } \\
\text { do curso em } 2002\end{array}$ & $\begin{array}{l}\text { Grade Curricular e Carga horária } \\
\text { do curso em } 2003\end{array}$ & $\begin{array}{c}\text { Grade Curricular e Carga horária } \\
\text { do curso em } 2004\end{array}$ \\
\hline $\begin{array}{l}\text { O contexto da Atenção à Saúde - } 40 \\
\text { horas }\end{array}$ & $\begin{array}{l}\text { UNIDADE I } \\
\text { O contexto da Atenção à Saúde - } 30 \\
\text { horas }\end{array}$ & $\begin{array}{l}\text { UNIDADE I } \\
\text { O contexto da Atenção à Saúde - } 20 \\
\text { horas }\end{array}$ \\
\hline $\begin{array}{l}\text { Determinação do processo saúde- } \\
\text { doença - } 20 \text { horas }\end{array}$ & $\begin{array}{l}\text { Sistema de Atenção à Saúde - } 30 \\
\text { horas }\end{array}$ & $\begin{array}{l}\text { UNIDADE II } \\
\text { Introdução à vigilância à saúde - } 60 \\
\text { horas }\end{array}$ \\
\hline $\begin{array}{l}\text { Planejamento e gestão do Trabalho } \\
\text { para a atenção à saúde - } 40 \text { horas }\end{array}$ & $\begin{array}{l}\text { Epidemiologia para os Serviços de } \\
\text { Saúde - } 40 \text { horas }\end{array}$ & $\begin{array}{l}\text { UNIDADE III } \\
\text { Vigilância à saúde no contexto das } \\
\text { ações e serviços - } 140 \text { horas }\end{array}$ \\
\hline $\begin{array}{l}\text { Decisões Clínicas baseadas em } \\
\text { evidências - } 20 \text { horas }\end{array}$ & $\begin{array}{l}\text { Métodos de Planejamento e Gestão } \\
\text { para o Trabalho em Saúde - } 40 \text { horas }\end{array}$ & $\begin{array}{l}\text { UNIDADE IV } \\
\text { O processo de trabalho das equipes de } \\
\text { saúde da familia - } 144 \text { horas }\end{array}$ \\
\hline $\begin{array}{l}\text { Metodologia da Investigação - } 40 \\
\text { horas }\end{array}$ & $\begin{array}{l}\text { Unidade Familiar, Trabalho, Ambiente } \\
\text { e Saúde - } 40 \text { horas }\end{array}$ & $\begin{array}{l}\text { Metodologia para elaboração do } \\
\text { trabalho monográfico - } 12 \text { horas }\end{array}$ \\
\hline $\begin{array}{l}\text { Saúde da Criança e do Adolescente - } \\
80 \text { horas }\end{array}$ & $\begin{array}{l}\text { UNIDADE II } \\
\text { Investigação em saúde: Aspectos } \\
\text { Metodológicos e Construção de uma } \\
\text { Proposta para o Serviço - } 45 \text { horas }\end{array}$ & $\begin{array}{l}\text { Trabalhos de Campo sob orientação - } \\
224 \text { horas }\end{array}$ \\
\hline Saúde da Mulher - 60 horas & UNIDADE III & MONOGRAFIA \\
\hline Saúde do Adulto - 60 horas & $\begin{array}{l}\text { Ciclo de vida: Saúde da Criança e } \\
\text { Adolescente - } 60 \text { horas }\end{array}$ & \\
\hline Saúde do Idoso - 20 horas & Saúde da Mulher horas- 60 & \\
\hline $\begin{array}{l}\text { Unidade Familiar, Trabalho, Ambiente } \\
\text { e Saúde - } 40 \text { horas }\end{array}$ & Saúde do Adulto - 40 horas & \\
\hline $\begin{array}{l}\text { Seminário para apresentação dos } \\
\text { trabalhos de conclusão - } 30 \text { horas }\end{array}$ & Saúde do Idoso - 20 horas & \\
\hline \multirow[t]{5}{*}{ MONOGRAFIA } & UNIDADE V & \\
\hline & $\begin{array}{l}\text { Tópicos especiais em saúde: Saúde } \\
\text { Mental na Familia, no Trabalho e na } \\
\text { Comunidade - } 25 \text { horas }\end{array}$ & \\
\hline & $\begin{array}{l}\text { Prevenção e Controle de Endemias - } \\
25 \text { horas }\end{array}$ & \\
\hline & Provisão de Medicamentos - 25 horas & \\
\hline & MONOGRAFIA & \\
\hline
\end{tabular}


As transformações pedagógico-estruturais dos três cursos de especialização promovidos pelo Programa de Interiorização do Trabalho em Saúde na Paraíba, emergiram a partir da constatação de que em relação a carga horária total dos cursos, o primeiro e o segundo mantiveram uma mesma carga horária de 450 horas, enquanto o terceiro sofreu um acréscimo de 150 horas, perfazendo um total de 600 horas de atividades pedagógicas.

Em relação ao número de disciplinas, a seqüência dos cursos apresentou um caminho inverso, se comparado ao item carga horária, pois, enquanto o PITs 1, oferecido em 2002 foi composto por onze disciplinas, o PITs 2 veiculado em 2003 apresentou doze disciplinas e o PITs - 3 foi formado por apenas seis disciplinas, divididas em quatro unidades programáticas. A divisão do curso em unidades foi originalmente iniciada em 2003 e mantida em 2004.

As mudanças mais significativas em relação aos aspectos pedagógico-estruturais dos três cursos promovidos pelo PITs na Paraíba foram registradas no âmbito da carga horária das disciplinas ofertadas ao longo dos três cursos. Enquanto nas duas primeiras versões a maior carga horária não ultrapassou oitenta horas, na terceira versão registraram-se disciplinas com 140 e 224 horas, respectivamente, Vigilância à saúde no contexto das ações e serviços e Trabalhos de campo sob orientação.

Quadro 02 - Produção acadêmica dos três cursos desenvolvidos pelo PITs na Paraíba

\begin{tabular}{|c|c|c|}
\hline Versão 2002 & Versão 2003 & Versão 2004 \\
\hline $\begin{array}{l}\text { Andrade MGN. Capacitação de } \\
\text { promotores de saúde: participação de } \\
\text { adolescentes do município de Santa } \\
\text { Inês/PB. }\end{array}$ & $\begin{array}{l}\text { Alves SLB. Abordando a violência } \\
\text { conjugal incidida sobre a mulher com } \\
\text { os agentes comunitários de saúde }\end{array}$ & $\begin{array}{l}\text { Tavares MLA. Perfil sócio-sanitário de } \\
\text { mães de crianças desnutridas no } \\
\text { município de Natuba/PB }\end{array}$ \\
\hline $\begin{array}{l}\text { Arcela NP, Paiva RBD. Situação } \\
\text { nutricional de crianças assistidas pela } \\
\text { pastoral da criança }\end{array}$ & $\begin{array}{l}\text { Melo NR. O trabalho dos agentes } \\
\text { comunitários de saúde do município de } \\
\text { Santa Inês/PB }\end{array}$ & \\
\hline $\begin{array}{l}\text { Cavalcanti KS, Aranha RCF. Estado } \\
\text { nutricional em crianças menores de } 5 \\
\text { anos da creche municipal Frei } \\
\text { Damião no município de Curral } \\
\text { Velho/PB }\end{array}$ & $\begin{array}{l}\text { Andrade RLMV. Dependência de } \\
\text { benzodiazepínicos na unidade de saúde } \\
\text { da familia Concórdia de Pedras de } \\
\text { Fogo/PB }\end{array}$ & \\
\hline $\begin{array}{l}\text { Silva JHO, Martins SSGH. Perfil } \\
\text { nutricional das crianças menores de } \\
\text { dois anos do município de Monte } \\
\text { Horebe/PB }\end{array}$ & $\begin{array}{l}\text { Frazão MGR. Dependência de } \\
\text { benzodiazepínicos: revisão bibliográfica }\end{array}$ & \\
\hline $\begin{array}{l}\text { Barros MAA, Targino RAA. face } \\
\text { oculta do Trauma: avaliação dos } \\
\text { acidentes domésticos na infância na } \\
\text { comunidade de Vila Nova/Cajá }\end{array}$ & $\begin{array}{l}\text { Soares INL. Uso indevido de } \\
\text { psicotrópicos em comunidade do } \\
\text { município de Diamante/PB }\end{array}$ & \\
\hline $\begin{array}{l}\text { Batista FAM. O processo de } \\
\text { implantação do PSF em João } \\
\text { Pessoa/PB: o caso do PSF São } \\
\text { José }\end{array}$ & $\begin{array}{l}\text { Araújo MCRF. Aspectos } \\
\text { epidemiológicos da esquistossomose } \\
\text { mansônica no município de } \\
\text { Alhandra/PB na área de abrangência } \\
\text { PSF 04/Oiteiro }\end{array}$ & \\
\hline $\begin{array}{l}\text { Câmara JEVS. Perfil de mortalidade } \\
\text { no município de Diamante/PB - } \\
1992 \text { a } 2001\end{array}$ & $\begin{array}{l}\text { Arruda IC. Agentes comunitários de } \\
\text { Saúde: potenciais e limitações }\end{array}$ & \\
\hline $\begin{array}{l}\text { Coelho AA, Monteiro MG. } \\
\text { Conhecendo o problema do lixo } \\
\text { doméstico na comunidade de } \\
\text { Barreiros, área rural do Município } \\
\text { de Cacimba de Dentro/PB }\end{array}$ & $\begin{array}{l}\text { Brandão DM. Efeitos de ações da } \\
\text { promoção da saúde sobre a } \\
\text { corporeidade e o bem estar de idosos }\end{array}$ & \\
\hline
\end{tabular}


Continuação

\section{Versão 2002}

Lima ES, Albuquerque OC. Determinantes e controle da hipertensão arterial em indivíduos hipertensos do povoado silvestre no município de Tavares/PB

Machado MLF, Gomes SKA. Implantação e implementação do Programa de atenção pré-natal no município de Nazarezinho/PB

Pessoa CLV, Cabral RWL. Atenção ao hipertenso no Programa de Saúde da Familia de São José de Princesa/PB

Zamora ARN, Morais MST. Programa de interiorização do trabalho em saúde no Estado da Paraíba: discurso de médicos e enfermeiros.

\section{Versão 2003}

Versão 2004

Dias KCCO. Fatores de risco para a osteoporose em mulheres idosas do município de casserengue/PB

Gomes SMA. Diagnóstico da saúde de crianças menores de dois anos de idade, matriculadas no serviço de puericultura do município de Santa Inês/PB

Medeiros AL. Tradições e fitoterapia: saberes que integram

Nóbrega LLR. Sexualidade na adolescência: discurso de adolescentes do município de Coxixola/PB

Paiva SG. Fatores determinantes do desmame precoce no município de Cacimba de Dentro/PB

Santana JS. Participação de homens no processo gestacional: um estudo com ênfase na divisão do trabalho doméstico

Silva CM. A adequação no uso de métodos contraceptivos na unidade de saúde da família de Nova Brasilia II Campina Grande/PB

Vieira PM. Resistência das mães à adesão ao aleitamento materno exclusivo
O nexo entre a produção acadêmica dos egressos dos três cursos de especialização com as mudanças pedagógico-estruturais verificadas nas três versões foi facilmente identificado no PITs - 2 (2003) com a inserção das disciplinas: Tópicos Especiais em Saúde, envolvendo a Saúde Mental na Família, no Trabalho e na Comunidade; Prevenção e Controle de Endemias e Provisão de Medicamentos com 25 horas-aula cada uma. Na prática, estas disciplinas influenciaram uma amostra significativa dos temas escolhidos para aprofundamento nas monografias. Verificou-se que das dezesseis monografias apresentadas em 2003, 13 (81,25\%) fizeram menção direta em seus resumos a influência das disciplinas na opção e desenvolvimento do tema escolhido para pesquisa.
Embora represente um fato de significativa importância o registro de apenas uma monografia haver sido apresentada no ano de 2004, não foi encontrado, na documentação pesquisada, nenhum indício do motivo para a ocorrência desse fenômeno. Para a formação do profissional vinculado ao PITs e sua reinserção no sistema de saúde local, a composição do trabalho acadêmico fundamentado nas bases conceituais, metodológicas e pedagógicas do Programa sob supervisão docente, possibilitou a superação dos problemas vivenciados em suas realidades de trabalho em bases científicas e sua conseqüente capacitação para realização de atividades de cuidados integrais de saúde à população, mediante ações de promoção, prevenção, diagnóstico e tratamento de acordo com 
os protocolos estabelecidos pelo Programa.

\section{CONSIDERAÇÕES FINAIS}

Os dados encontrados permitem asseverar que houve uma correspondência direta entre as mudanças pedagógico-estruturais verificadas nas três versões do Programa de Interiorização do Trabalho em Saúde na Paraíba e a opção pelo tema da produção acadêmica dos egressos desses cursos.

Os dados apresentados, também conformam a base para a realização de futuras investigações sobre a efêmera passagem do Programa de Interiorização do Trabalho em Saúde, notadamente sobre seus aspectos político-conceitual, político-pedagógico e político-metodológico, na tentativa de fazer emergir as razões que levaram a não reedição de uma iniciativa de tamanha importância para o processo de formação de força de trabalho em saúde.

Sugere-se, por fim, o desenvolvimento de pesquisas acadêmicas sobre o impacto destas produções no âmbito da saúde dos locais de origem dos recursos humanos qualificados nos três cursos ministrados e a conseqüente publicação de seus resultados.

\section{REFERÊNCIAS:}

1. Santana MNGS. Política de recursos humanos para o SUS e a política de formação de profissionais em enfermagem. In: Anais do $5^{\circ}$ Seminário Nacional de Diretrizes para o Ensino de Enfermagem; 2001 julho 0407; São Paulo: CUSC; 2001. p. 69-81

2. Ministério da Saúde (BR). Secretaria Executiva. Coordenação Geral de Desenvolvimento de Recursos Humanos para o SUS. A investigação sobre recursos humanos em saúde. Brasília; 1993.

3. Ministério da Saúde (BR). Secretaria Nacional de Assistência à Saúde. ABC do SUS: Doutrinas e princípios. Brasília; 1990.

4. Souza MF. A enfermagem reconstruindo sua prática: mais que uma conquista no PSF. Rev Bras Enferm 2000 Dez; 53(n. esp):25-30.

5. Moura ADA, Lima GG, Braquehais AR. Programa de interiorização do Trabalho em Saúde - PITs: uma estratégia que deu certo. Rev RENE. 2005 Mai/Ago;6(2): 47-54.

6. Ministério da Saúde (BR). Programa de Interiorização do Trabalho em Saúde: concepções, seleção e lotação de equipes. Brasília: Secretaria de Políticas de Saúde, Out. 2001.

7. Ministério da Saúde (BR). Conselho Nacional de Saúde. Diretrizes e normas regulamentadoras de pesquisa envolvendo seres humanos. Resolução n.. ${ }^{\circ}$ 196, de 10 de outubro de 1996. Brasília; 1996

Cogitare Enferm 2008 Jan/Mar; 13(1):96-102 